\title{
Traduire
}

Revue française de la traduction

227 | 2012

Éco, socio, philo... \& co

\section{Traduction multimédia et voix régionales : la version française du film Respiro d'Emanuele Crialese}

Antonino Velez

\section{(2) OpenEdition \\ Journals}

\section{Édition électronique}

URL : http://journals.openedition.org/traduire/496

DOI : $10.4000 /$ traduire.496

ISSN : 2272-9992

Éditeur

Société française des traducteurs

\section{Édition imprimée}

Date de publication : 15 décembre 2012

Pagination : 101-116

ISSN : 0395-773X

\section{Référence électronique}




\section{Traduction multimédia et voix régionales : la version française du film Respiro d'Emanuele Crialese}

\section{La traduction multimédia}

Dans son essai Translation and mass communication, Dirk Delabastita (1989 : 193-218), définit la notion de base de film translation comme une série d'opérations visant à transposer un film d'un système culturel d'origine dans un autre. Selon la théorie de Delabastita, le texte d'un film est le résultat de l'interaction entre des canaux (acoustiques et visuels) et des codes (y compris les variantes linguistiques ou paralinguistiques comme l'intonation).

Le texte audiovisuel représente, en effet, un type de texte à part, où la sphère sonore (qui comprend, outre les dialogues, les sons et les bruits, c'est-à-dire la bande son) et la sphère visuelle se combinent pour créer un texte complexe multicodes - un texte multimédia très articulé - dont la transposition dans une langue différente de l'originale peut poser de nombreux problèmes.

Partant, les études critiques sur la traduction multimédia, qui opère sur des textes complexes constitués par des langages différents ou par des systèmes sémiotiques qui agissent contemporainement, doivent opérer une analyse sur la base de l'effet de sens général qu'elle produit (Bollettieri, $2000:$ 432).

En dépit du fait que le langage cinématographique se base sur l'interrelation entre des codes différents - visuels, verbaux et sonores - le code verbal est le seul qui peut être modifié et manipulé dans le passage d'une langue à une autre.

La traduction cinématographique doit être à même de recréer dans la langue d'arrivée une trame d'oralité artificielle qui reflète l'oralité du texte de départ. Cette opération de transfert est fortement conditionnée par les nombreux obstacles engendrés par la coprésence de plusieurs codes.

La traduction des dialogues ne doit pas être pensée uniquement d'un point de vue linguistique, mais doit tendre à restituer le sens global de l'œuvre dans le respect des limites impo- 
sées par les images. En outre, le traducteur se trouve face à un dialogue de film sous forme de texte écrit qu'il doit transposer en une conversation parlée ; il a, par conséquent, la tâche difficile de reproduire dans la langue d'arrivée un parlé simulé, c'est-à-dire joué, tout en gardant à l'esprit des scènes pleines de gestes, de sons et d'images. Le langage cinématographique n'est pas spontané mais adapté puisqu'il est soumis à une série de processus techniques (montage, postsynchronisation, etc.) et il est en général contraint par la nécessité d'être compréhensible par un public aussi vaste que possible. II s'agit d'un langage oral artificiel, écrit pour être lu de manière à sembler authentique, alors qu'en fait il n'a pas grand-chose à voir avec l'immédiateté de l'oralité (Paolinelli, 2005 : 8-9).

Les films et leurs versions doublées constituent un bon exemple de traduction multimédia : ce sont des textes parallèles dont il est relativement facile d'évaluer le " fonctionnement " sur la base de leur perception par le public dans les deux cultures.

Aborder le problème de la traduction pour le cinéma signifie mettre constamment en rapport les dialogues avec le sens qu'ils véhiculent à travers les images et la bande son. Le dialoguiste doit étudier les rapports existant entre les différentes composantes du langage cinématographique pour permettre à ceux qui suivent la version doublée, même dans un contexte culturel différent, de pouvoir jouir d'un produit correctement recodé. Une théorie de la traduction, à même de faire face aux problèmes du multimédia, doit prendre en considération les processus qui se définissent d'habitude avec le terme d'" adaptation " plutôt que de traduction.

L'adaptation est une réécriture qui doit tenir compte de différents facteurs : tout d'abord les intentions de l'auteur original. Pour cette raison les adaptateurs-dialoguistes sont considérés, à juste titre, comme de véritables auteurs ayant toute légitimité à apporter des variations dans la traduction (Le Nouvel, 2007 : 43-47).

La notion d'équivalence (1), introduite au début des années soixante pour définir la traduction en général, est aujourd'hui utilisée pour décrire la nature et le type des correspondances établies entre le texte original et le texte traduit. Les spécialistes des Translation Studies ont montré que la notion d'équivalence, comme celles de fidélité ou de traduction littérale, est déterminée par le contexte historique, social et idéologique, et par la perception personnelle du traducteur et/ou du dialoguiste-adaptateur (Gentzler, 2001).

L'objectif d'une traduction équivalente est de produire le même effet que l'original, y compris en utilisant des mots différents le cas échéant (Perego, 2005 : 41-43) ou en ayant recours à des éléments plus appropriés au niveau culturel, de manière à faciliter la compréhension par

(1) Mentionnons à titre d'exemples les ouvrages de Vinay et Darbelnet (Stylistique comparée du français et de I'anglais : Méthode de traduction, Paris, Didier, 1968) et Catford John C. (A Linguistic Theory of Translation: an Essay on Applied Linguistics, London, Oxford University Press, 1965). 
le public de la langue d'arrivée. Au sujet de la traduction cinématographique, Herbst déclare :

The wording of the original does not matter, what matters is that all plot-carrying elements of a scene should be translated and that some kind of equivalence of the characters' personality on screen should be maintained (Herbst, 1996 : 105)(2).

Il propose ainsi une approche pragmatique de la traduction des films, car il estime que le but devrait en être l'obtention du même effet dans la version doublée. Si le film contient des dialectes, la traduction devient plus compliquée.

D'autres études sur la traduction(3) (applicables à l'audiovisuel) adoptent une stratégie de traduction qui se base sur l'adéquation et l'acceptabilité de la traduction elle-même ; selon cette théorie, le produit de la traduction doit essentiellement adhérer aux valeurs et aux références de l'original (adéquation) et tenir compte des valeurs linguistiques et culturelles du polysystème de destination (acceptabilité).

La traduction pour le cinéma ne peut pas être jugée exclusivement sur la base de la fidélité au texte d'origine ou de sa réussite esthétique. Une bonne adaptation d'un film doit être avant tout efficace : on peut être infidèles du point de vue linguistique, mais fidèles du point de vue cinématographique, ou efficaces et crédibles (Cipolloni, 1997).

\section{Le film de Crialese}

Respiro, tourné pendant l'été de 2001 dans l'île de Lampedusa (Sicile), obtint en 2002 un grand succès au festival de Cannes(4). L'accueil de ce film, qualifié par la critique de " merveilleux produit du cinéma italien ", acclamé et ovationné par le public et la critique, a dépassé les attentes. Respiro a été distribué dans le monde entier et il a remporté un grand succès public, surtout en France et en Allemagne(5). Un mangiadischi (tourne-disques portatif pour 45 tours) qui joue une chanson de Patty Pravo, La bambola, permet de situer le film dans le décor d'une Lampedusa de la fin des années soixante. Les interprètes principaux sont Vincenzo Amato et une exceptionnelle Valeria Golino. II s'agit d'une œuvre étrange et difficile avec une histoire presque inexistante qui veut surtout mettre en évidence le climat d'oppression qui règne dans

(2) "La formulation de l'original n'a pas d'importance ; ce qui est important, c'est que dans chaque scène tous les éléments intervenant dans la progression de l'intrigue soient traduits et qu'une certaine équivalence de la personnalité des personnages soient conservée à l'écran. "

(3) Voir à ce propos la théorie des polysystèmes d'Even-Zohar et les études de Gideon Toury.

(4) Prix de la Semaine de la Critique au 55e Festival de Cannes ; primé aux festivals de Toronto et d'Athènes et Prix Duel 2003. Valeria Golino a remporté le "Ruban d'argent " de Taormine (meilleure actrice), pour son rôle dans Respiro.

(5) http://www.isoladilampedusa.it/articoli/respiro.htm [dernière consultation le 14/03/2012] 
l'île. La trame, qui n'est pas linéaire, est en mouvement continu et nous rappelle l'ondulation de la mer. L'île devient un monde de rêve, où la cruauté des hommes (des représentants du sexe masculin) laisse la place à l'onirisme et aux fantasmes, où le personnage incarné par Valeria Golino, Grazia, se sent libre de respirer (respiro = souffle) seulement quand elle plonge dans la mer, devenant une sorte de "Vénus sortie des ondes, porteuse de toutes les pulsions, de toutes le passions, de tous les fantasmes " (Serroy, 2006 : 236-237).

Le film révèle l'originalité et le talent du jeune metteur en scène Emanuele Crialese, romain mais d'origine sicilienne, qui non seulement fait entendre le dialecte de Lampedusa, mais laisse aussi s'exprimer les visages des habitants, les bruits et le " souffle " de l'île. L'eau de la mer qui baigne cette dernière devient métaphore d'un refuge où Grazia peut exprimer sa liberté niée(6).

Respiro est un film exigeant et en même temps difficile à cause des dialogues en dialecte de Lampedusa(7), sa narration saccadée et ses répliques laconiques. C'est un film, également, équilibré et évocateur qui procède par petites touches descriptives très justes. Cette peinture de l'île de Lampedusa ne se contente pas de refléter l'image typique des cartes postales, avec le soleil, le sable et la mer, mais réussit à harmoniser le tout, donnant corps à une réalité intrigante, à plusieurs facettes ; un mix de solidarité, de dur labeur, de complicité et de cohabitation entre la loi et l'illégalité, les rivalités entre bandes de jeunes, la compassion familiale et la dévotion religieuse, la violence et le machisme. La plus grande réussite de Crialese est, à notre avis, d'avoir surtout su éviter tout folklore(8).

Le film s'ouvre avec la scène d'une bande de jeunes adolescents, à la peau brulée par le soleil, sauvages et primitifs, qui chassent des petits oiseaux et puis les mangent, dans un milieu parsemé de maisons délabrées et abandonnées ou dans d'amples espaces filmés en plongée(9). Ils se déplacent en troupeau, comme une meute, parlent en dialecte et regardent avec mépris et incrédulité tout étranger (comme ce sera le cas du carabinier).

(6) http://movies-home.blogspot.com/2008/05/respiro-emanuele-crialese.html [dernière consultation avril 2012]

(7) Le linguiste sicilien Giovanni Ruffino dans son étude I dialetti di Lampedusa e Linosa, con particolare riguardo alla terminologia marinaresca (Biblioteca del Centro di studi filologici e linguistici siciliani, $2^{\mathrm{a}}$ serie, 2, 1977), décrit le dialecte parlé à Lampedusa comme un dialecte indéfinissable du point de vue de l'aire et qui ne peut pas s'inscrire dans un schéma précis de classification des parlers siciliens. On peut parler en général d'un dialecte essentiellement de la Sicile occidentale, riche en traits de la Sicile centrale (et notamment d'Agrigente), avec certains éléments particuliers des dialectes de la Sicile orientale et notamment de la zone de Messine.

(8) DZIGA CACACE, E ora facciamo tutti un bel respiro, di E. Crialese, Italia/Francia 2002, in Divine Divane Vision (lena Videns 02/03) - 27, Cinema Ducale, Milano, 01/09/2002 [Document en ligne]. http://www.carmillaonline.com/archives/2011/09/004019.html [dernière consultation le 12/04/2012].

(9) http://www.cinemovie.info/Respiro_scheda.htm [dernière consultation le 14/04/2012]. 


\section{Méthode d'analyse}

Nous appliquerons une analyse contrastive qui compare la version originale du film à la version doublée en français. Ne possédant pas le script officiel, nous avons retranscrit les dialogues directement à partir du film(10).

Notre analyse suivra un parcours précis : en partant de la subdivision du film en 16 macroscènes (avec la transcription des dialogues dans les deux langues), nous allons nous arrêter sur certains aspects de la version doublée en français pour mettre en évidence les différences de registre linguistique (surtout au niveau des idiolectes et des sociolectes). Nous verrons que pour résoudre les problèmes posés par les éléments dialectaux, le dialoguiste a recouru en français au langage familier et à l'argot afin de mieux caractériser les personnages. Au cours de notre étude nous indiquerons par VO le parlé original et par VD la version doublée (nous ferons référence à la version sous-titrée, VS, pour des cas particuliers). Chaque microanalyse sera introduite par une brève introduction qui mettra tour à tour en évidence l'approche du traducteur dialoguiste face aux problèmes d'adaptation. Le personnage qui parle sera marqué en gras ainsi que les mots objet d'une remarque particulière.

\section{La version française}

Le rapport entre traduction et adaptation est évident dès le choix du titre. Le titre d'un film peut être parfois un élément important pour choisir la vision d'un film de la part du public. Pour la version française, le titre a été conservé dans la langue d'origine(11), ce qui est en soi significatif et porteur d'un message pour le public de la langue d'arrivée (Cipolloni, 1997 : 102). Rappelons, en outre, que dans la plupart des cas le titre n'est pas choisi par le dialoguiste-adaptateur, mais par le bureau de marketing et de diffusion de la maison de production (Paolinelli, 2005 : 52). Par ailleurs, le titre original du film aurait dû être différent puisque le réalisateur avait choisi Oscià(12), un mot du dialecte de Lampedusa, utilisé pour saluer quelqu'un avec

(10) II existe des conventions scientifiques sur la façon de mettre les dialogues dans une forme écrite. La fonction la plus significative de la transcription consiste dans la perception et dans la segmentation des messages verbaux, qui doivent être combinés avec ce qui se passe dans le film et, par conséquent, tenir compte des codes sonores, visuels et dynamiques. II s'agit d'attribuer correctement les mots et les autres expressions verbales et paraverbales en indiquant les pauses, les éléments prosodiques, les interjections et en cherchant à préciser quand et comment les répliques se superposent. [cf. R.M. BOLLETTIERI BOSINELLI, C. HEISS, M. SOFFRITTI, BERNARDINI, ouvr. cité, p. 185].

(11) Dans d'autres pays on a préféré des titres différents: Grazia's Island (pays anglophones), La Isla de Grazia (pays hispanophones), Lampedusa (en Allemagne).

(12) Aujourd'hui oscià est le nom d'une manifestation musicale qui a lieu depuis de nombreuses années à Lampedusa, au mois de septembre, sous la direction du célèbre chanteur italien Claudio Baglioni. 
affection et qui signifie " mon souffle ", " ma joie "(13). C'est le producteur Domenico Procacci qui a conseillé Respiro, titre qui évoque tant la possibilité de respirer que le sentiment de liberté et d'harmonie intérieure et qui est compréhensible par un plus vaste public. Le choix français du maintien du titre original est probablement dicté par la volonté de mettre en évidence son caractère de " film d'essai ".

\section{- Macroscène 11, minutage 56:14}

II s'agit d'une scène très tendre entre Grazia et son fils Filippo, au cours de laquelle ce dernier, après l'avoir invitée à dormir avec lui, chante à sa mère un couplet de la chanson La bambola. Le public français doit comprendre la scène uniquement sur la base de ses connaissances(14) et de son implication émotionnelle dans le film.

\begin{tabular}{|l|l|}
\hline VO & VD \\
\hline Filippo : dormi cu mme. & Filippo : dors avec moi. \\
$\begin{array}{l}\boldsymbol{D} \text { Tu mi fai girar, tu mi fai girar, } \\
\text { come fossi una bambola. }\end{array}$ & $\begin{array}{l}\text { CTu mi fai girar, tu mi fai girar } \\
\text { come fossi una bambola. }\end{array}$ \\
\hline
\end{tabular}

\subsection{Les variétés linguistiques}

La sociolinguistique prend en considération toute une série de caractéristiques de la langue parlée particulièrement difficiles à reproduire, telles que les variétés régionales (diatopie), la classe sociale (diastratie), le niveau de formalité du langage et les problèmes dus à l'adoption d'un certain registre (diamésie). L'équivalence parfaite de la situation sociolinguistique d'un film est impossible. Respiro abonde en expressions siciliennes qui parfois n'ont même pas d'équivalence en italien et en tout cas n'auraient pas la même force expressive. C'est le cas de l'expression en sicilien c'è i moriri (à la lettre : « il y a de quoi mourir ") prononcé par Filippo, le fils de Grazia, dans la macroscène 13, qui en VD devient " c'est pas croyable ! ", perdant ainsi toute la couleur de la phrase sicilienne (un humour noir qui combine la mort avec un sentiment d'incrédulité) :

\begin{tabular}{|l|l|}
\hline V0 & VD \\
\hline Filippo : andatevene a casa vostra. & Filippo : vous deux là, rentrez chez vous. \\
Ancora cca siti? & Encore là ? Dégagez ! \\
Cu ccu staiu parlannu cu muru, io? & À qui je parle moi ? À un mur ou quoi ? \\
C'è i moriri, va'! & C'est pas croyable ça! \\
\hline
\end{tabular}

(13) B. SORRENTINI, Intervista ad Emanuele Crialese, in Altro Cinema [Document en ligne] http://www.altrocinema.it/archivio/archivi/respiro.htm, [dernière consultation le 05/04/2012].

(14) Le public français moins jeune est censé connaître cette chanson par le truchement de la version française, du même titre, chanté à la fin des années soixante par Dalida. II existe même une version en duo où Patty Pravo et Dalida chantent ensemble La Bambola mélangeant couplets en italien et en français. http://www.youtube.com/watch?v=AqoQBzbo6YY\&NR=1\&feature=endscreen (cf. youtube 10/10/12). 
Lorsque les conflits de classes transparaissent également à travers les variétés linguistiques, le dynamisme sociolinguistique risque d'être perdu si, dans la version doublée, tous les personnages parlent de la même manière. La difficulté de restituer dans une autre langue les registres bas, les variations ou les fautes de prononciation et les expressions familières est le plus grand des défis pour l'adaptateur-dialoguiste (Paolinelli, 2005 : 18-21).

Le dialecte est difficile, voire impossible, à reproduire : il ne peut exister de véritable analogie entre un dialecte et le langage standard. L'alternative consiste à proposer un langage particulier en travaillant sur le texte plutôt que sur l'accent, c'est-à-dire en intervenant sur le plan lexical et morphosyntaxique de façon à recréer dans la langue cible des effets proches de ceux de la langue source (Bollettieri, 2000 : 62-68).

Quand on traduit un dialogue il est fondamental de décider " comment " dire les choses et pas seulement " quoi » dire. Parfois même le plus simple des éléments (une conjonction, par exemple) doit être traduit en tenant compte du personnage et de la situation ou, évidemment, du mouvement des lèvres (Paolinelli, 2005 : 59-62). L'absence de transposition d'un des marqueurs de l'oralité, des expressions familières et des vulgarités, des accents et des fautes de prononciation risque de miner la compréhension du public de langue cible.

Le dialoguiste doit être particulièrement habile dans la reconstruction des connotations d'une langue avec certaines caractéristiques ; il doit faire des choix lexicaux précis et avoir recours, s'il est nécessaire, à tous les moyens possibles pour rendre le clivage linguistique original dans la version doublée. Malheureusement, souvent, les difficultés rencontrées amènent le dialoguiste à aplatir la langue d'arrivée dans ce qu'on appelle en italien le doppiaggese. L'objectif principal doit donc être de donner le maximum de vivacité aux dialogues et de distinguer les différents niveaux de langue des personnages (Bollettieri, 2000 : 46).

\subsection{La langue du film : niveau syntaxique et linguistique}

Une des caractéristiques du parlé original de Respiro se base sur l'élément linguistique qui marque les différents personnages en mettant en évidence tour à tour leur provenance géographique, leur appartenance à un groupe ou à une classe sociale, leur niveau d'instruction, etc.

Le film est surtout parlé en dialecte de Lampedusa (hormis quelques dialogues en italien standard), un parler diatopiquement marqué, qui place chaque personnage dans un contexte sociolinguistique bien défini. En outre, le niveau syntaxique ainsi que le niveau lexical sont des contraintes inévitables liées aux personnages (hommes, femmes et enfants) de basses couches sociales. Les phrases sont brèves, les dialogues laconiques mais denses de signification, sans tournures complexes. La langue utilisée dans le film se différencie de l'italien standard également par l'emploi de certains temps verbaux, notamment le passé simple, et par le bouleversement de l'ordre syntaxique des phrases, typique du sicilien. En voici deux exemples : 


\section{- Macroscène 13, minutage 1:02}

Après la disparition de Grazia, son mari commence les recherches. Dans la VD, le passé simple, temps verbal fréquemment utilisé en sicilien à l'oral même pour exprimer une action qui vient de se terminer, est traduit par le passé composé.

\begin{tabular}{|l|l|}
\hline V0 & VD \\
\hline Pietro : a truvasti? & Pietro : tu l'as trouvée ? \\
\hline Pasquale : no! & Pasquale : non. \\
\hline Pietro : dove la cercasti? & Pietro : t'as cherché où ? \\
\hline Pasquale : a punta i l'Albero e a Punta du Suli. & Pasquale : à la Pointe et à l'Arbre du soleil. \\
\hline $\begin{array}{l}\text { Pietro : tu và a Cala Creta, } \\
\text { noi andiamo dall'altra parte. }\end{array}$ & $\begin{array}{l}\text { Pietro }: \text { continue à chercher. } \\
\text { On va de l'autre côté. }\end{array}$ \\
\hline
\end{tabular}

Cette scène met en évidence une autre contrainte du doublage : la traduction de noms de localités et de lieux géographiques. En général, si ceux-ci se réfèrent à des lieux que le public de la langue d'arrivée ne peut pas aisément reconnaître, deux solutions sont possibles : soit on a recours à un autre nom géographique, soit on élimine le nom carrément (Baccolini, 1994 : 90-91). Dans la scène ci-dessus le premier nom de lieu a été maintenu (et traduit plus ou moins correctement !) tandis que le second a été supprimé. On peut ici s'interroger sur la logique du choix du dialoguiste, car il s'agit de deux lieux totalement inconnus du public français.

Parmi les stratégies adoptées par le doublage français, on peut remarquer une tendance à la normalisation et à la standardisation du langage où toute différence sociolinguistique est aplatie et éliminée (Pavesi, 2005 : 55). La version doublée perd par conséquent toute connotation liée au dialecte. La reconstruction du milieu sicilien, pour le public français, se borne au décor et aux images.

Le français adopté dans le doublage est parsemé de formes familières, argotiques et parfois vulgaires, produisant de ce point de vue un contenu linguistique fidèle à l'original.

Pour rendre compte de la variation diastratique et pour reproduire en quelque sorte l'effet de l'original, le dialoguiste a recours à d'autres marqueurs typiques de la langue familière. II utilise des tournures typiques de la langue parlée à travers des choix morphosyntaxiques et lexicaux qui créent dans la langue d'arrivée une certaine correspondance avec la langue de départ.

Un élément typique du français parlé est l'interjection " hein ", généralement placée à la fin d'une phrase, au lieu de "n'est-ce pas ", utilisé pour impliquer l'interlocuteur en lui demandant de confirmer. En voici un exemple (il s'agit ici d'une compensation, l'original étant en italien standard) :

- Tiré de la Macroscène 9, minutage 43:28

\begin{tabular}{|l|l|}
\hline VO & VD \\
\hline Carabiniere : Bello, vero? & Carabinier : C'est magnifique, hein ? \\
\hline
\end{tabular}


L'interjection " ouais ", également familière, apparaît aussi dans certaines répliques du film(15). Le dialoguiste a cherché à travers l'emploi diffus de marqueurs de la langue française orale (élision de certaines voyelles ou certaines interjections, par exemple) à se rapprocher du langage parlé spontané des habitants de l'île de Lampedusa.

\section{- Macroscène 6, minutage 25:45}

Grazia également ne s'exprime qu'en dialecte. Pier Luigi, le carabinier (Elio Germano), qui vient du nord de l'Italie, est le seul personnage du film à s'exprimer en italien. Cette différence marque aussi le décalage et la distance existant entre les habitants de l'île et le reste de l'Italie incarné par l'homme de la loi. Malheureusement cette distinction, très importante dans le film, n'est pas reproduite en français. De plus, le langage du carabinier en italien est très formel. II dit par exemple "sprovvista di documenti ", qui est simplement transcrit : " vous n'avez pas vos papiers ". Cette traduction produit, au niveau mésostructurel et monostructurel, une réduction d'effet et un affadissement (Hewson, 2011 : 25-27) :

\begin{tabular}{|l|l|}
\hline V0 & VD \\
\hline $\begin{array}{l}\text { Carabiniere : buongiorno signorina. } \\
\text { Favorisca i documenti per favore. }\end{array}$ & $\begin{array}{l}\text { Carabinier : bonjour mademoiselle. } \\
\text { Je peux voir vos papiers, s'il vous plaît ? }\end{array}$ \\
\hline Marinella : li ho lasciati a casa & Marinella : ils sont à la maison. \\
\hline $\begin{array}{l}\text { Carabiniere : Cioè lei sta circolando } \\
\text { con due passeggeri a bordo ed è sprovvista } \\
\text { di documenti relativi al ciclomotore e personali? }\end{array}$ & $\begin{array}{l}\text { Carabinier : non seulement vous circulez avec deux } \\
\text { passagers à bord et en plus, vous êtes en train } \\
\text { de me dire que vous n'avez pas vos papiers ? }\end{array}$ \\
\hline Marinella : lei è nuovo vero? & Marinella : vous êtes nouveau ici ! \\
\hline Carabiniere : sì, sono in servizio da ieri & Carabinier : je suis en service depuis hier. \\
\hline Grazia : benvenuto! & Grazia : bienvenue chez nous ! \\
\hline $\begin{array}{l}\text { Carabiniere : grassie(16). Dai...per stavolta lascio } \\
\text { andare.. però uno di voi deve andare a piedi, } \\
\text { che senò chi vi vede cosa pensa? }\end{array}$ & $\begin{array}{l}\text { Carabinier : merci... Pour cette fois... } \\
\text { je ferme les yeux. Mais l'un de vous doit } \\
\text { continuer à pied, sinon, j'aurai l'air de quoi ? }\end{array}$ \\
\hline Grazia : scinni Filì. & Grazia : descends, Filippo. \\
\hline $\begin{array}{l}\text { Filippo : pecchè devo scendere? Non fai sfaventare } \\
\text { nessuno con questa paletta e questa pistola, }\end{array}$ & $\begin{array}{l}\text { Filippo : pourquoi moi ? } \\
\text { Tu me fais pas peur avec ton espèce } \\
\text { pe... pezzo di sbirro. }\end{array}$ \\
\hline Grazia : ti dissi di scendere Filì.... Scusate! Grazie! ton pistolet. Poulet ! \\
\hline \begin{tabular}{l} 
Carabiniere : niente dai... dai, andate su! \\
Grazia : allons, on t'a dit de descendre, Filippo! \\
\hline
\end{tabular} & Désolée. Merci. \\
\hline
\end{tabular}

(15) Cf. macroscène 8 , minutage $37: 16$, dans cette même étude.

(16) Dans le film on ne dit pas explicitement d'où vient le carabinier mais le spectateur italien peut s'en faire une idée à partir de la prononciation du mot grazie /grassie/ : il ne peut qu'être du nord de l'Italie. En effet, ce type de prononciation (assibilation d'une affriquée) est un trait phonétique typique du bas Piémont, de la Ligurie, de l'Émilie, de la Vénétie, du Frioul et de la Lombardie (trait populaire). 
D'autre part la VD adhère à la V0 dans le choix des termes familiers. C'est le cas de "poulet " qui traduit le mot péjoratif italien sbirro pour policier. Le synchronisme labial dans le doublage empêche le recours à un mot argotique pour traduire pistola qui devient " pistolet " en VD tandis qu'en VS on peut se permettre l'emploi de "flingue " qui respecte davantage le ton de la scène. Dans cette scène il faut noter en outre le bégaiement de Filippo(17) (pe...pezzo di sbirro) et ses fautes de prononciation (" sfaventare " au lieu de "spaventare ", faire peur) qui n'ont pas d'équivalence dans le doublage. Et encore une différence incompréhensible dans cette scène est le fait que la mère, Grazia, appelle son fils Filippo en italien par le diminutif " Filì " (avec accent sur le i final) et le dialoguiste français n'a pas voulu reproduire la même abréviation dans la VD, allongeant ainsi la réplique.

- Macroscène 2, minutage $08: 12$

Les protagonistes de cette scène sont un groupe de jeunes, parmi lesquels Pasquale et Filippo, aux prises avec une sorte de tatouage, fait au stylo, par deux amies rencontrées dans le cours principal. On traduit en français l'expression familière che rompiscatole par " se faire chier ", appartenant à un lexique vulgaire. En outre, dans cet extrait, on remarque une caractéristique de la langue sicilienne, relative à la façon d'exprimer la négation : il s'agit d'un son presque imperceptible $n z u$, un clic dental qui représente une négation non lexicale ; la langue française ne dispose d'aucun équivalent (l'italien non plus d'ailleurs) :

\begin{tabular}{|l|l|}
\hline VO & VD \\
\hline $\begin{array}{l}\text { Filippo : u gabbianu ci sta fannu... tuttu } \\
\text { spinnacchiatu, senza penni... Tuttu spinnatu }\end{array}$ & Filippo : Oh ! La mouette, toute pelée ! \\
\hline Pasquale : e zittiti... va ddà... & Pasquale : Filippo tais-toi ! Dégage ! \\
\hline Filippo : oh, ma chi rumpiscatuli cà! & Filippo : oh et puis on se fait chier \\
\hline Amico : ou ti zzitti? & Un ami : oh ferme-la \\
\hline Pasquale : andiamo via noi soli? & Pasquale : on va faire un tour tous les deux ? \\
\hline Alessandra : nzù & Alessandra : [elle hoche la tête en signe de refus] \\
\hline Pasquale : sei bella & Pasquale : tu es trop belle. \\
\hline Alessandra : grazie & Alessandra : merci. \\
\hline Pasquale : me lo dai un bacio? & Pasquale : tu veux bien m'embrasser ? \\
\hline Alessandra : no & Alessandra : non \\
\hline Filippo : u baciu a tia t'avi a dari chi non ti lavi mai? & $\begin{array}{l}\text { Filippo : t'embrasser alors que tu chlingues à des km. } \\
\text { Tu te laves jamais ? Elle t'embrassera jamais ! } \\
\text { Non ti nni duna baciu... No no no no }\end{array}$ \\
\hline
\end{tabular}

(17) Que nous avons signalé à l'écrit par trois points de suspension. 
- Macroscène 8, minutage 37:16.

Dans cet échange laconique de répliques, Marinella demande une cigarette au carabinier.

\begin{tabular}{|l|l|}
\hline V0 & VD \\
\hline Marinella : ciao. & Marinella : salut. \\
\hline Carabiniere : ciao. & Carabinier : salut. \\
\hline Marinella : hai una sigaretta? & Marinella : t'as une cigarette ? \\
\hline Carabiniere : S-si! & Carabinier : ouais !(18) \\
\hline Marinella : hai da accendere? Grazie! & Marinella : du feu aussi ? Merci. \\
\hline Carabiniere : prego! & Carabinier : pas de quoi ! \\
\hline$[\ldots]$ & {$[\ldots]$} \\
\hline Amica : e allora, chi ci dicisti? & Amie : alors, qu'est-ce qu'il t'a raconté ? \\
\hline Marinella : mi ha dato una sigaretta. & Marinella : il m'a donné une cigarette. \\
\hline Amica : e basta? & Amie : et puis ? C'est tout ? \\
\hline Marinella : e basta! & Marinella : ouais, c'est tout ! \\
\hline
\end{tabular}

La traduction du mot sigaretta rend compte des différences de contraintes entre la VD et la VS. Dans le premier cas, c'est le synchro labial qui commande et, par conséquent, on traduit en français standard " cigarette ". Dans la VS c'est la brièveté qui l'emporte et on traduit avec le terme familier « clope".

\subsection{Les gros mots}

Il est intéressant de faire quelques remarques à propos de la façon dont sont gérées les grossièretés qui abondent dans le film. La traduction des gros mots est un exemple significatif des choix de transfert du lexique d'une langue dans l'autre en phase de doublage, qui revient souvent à en atténuer le ton à cause des difficultés que leur traduction comporte. L'usage des grossièretés, fréquentes dans le film que nous étudions, tend à reproduire la façon de parler informelle et quotidienne d'une partie de la population, qui utilise des termes vulgaires, allant de la légère imprécation à des expressions plus injurieuses et blessantes. L'emploi de mots vulgaires dans ce contexte a cependant une signification bien précise qui ne doit pas être négligée dans la traduction.

Quelquefois, certaines expressions trop colorées ont été atténuées ou rendues de façon littérale, s'agissant de formules improvisées(19), créées, au moment de l'action, par les personnages dans la V0, et donc ayant difficilement un équivalent dans la langue d'arrivée.

(18) Cf. macroscène 9 minutage 43:28 dans cette étude.

(19) Selon la définition de Gregory et Carrol (Language and Situation. Language Varieties and their Social Contexts, London, Routledge, 1978: 42) : "written to be spoken as if not written". 


\section{- Macroscène 6, minutage 27:43}

Nous proposons ci-dessous un extrait de la macroscène 6 , où, au cours d'une dispute entre Grazia et une autre femme, on utilise l'expression : "testa di pane duru ". Cette formule en sicilien signifie littéralement, " tête de pain dur " et désigne une personne têtue. Dans ce contexte, la réplique de la femme offensée (" ma chi ti credi Patty Pravo ") suggère plutôt l'idée d'une "personne inutile ", en opposition avec celle qui croit être une vedette. La VD traduit cette réplique par " tête de morue ". C'est une trouvaille géniale du dialoguiste français qui met cette expression dans la bouche de Grazia au moment où la scène se passe dans une usine de conserve de poisson (femmes et enfants y travaillent). La version française gagne d'une part en signification avec le jeu de mots sur le poisson, même si elle perd, de l'autre, la référence culturelle à la chanteuse Patty Pravo, dont on entendait au début du film La bambola: le nom de l'artiste italienne est omis en français et remplacé par un neutre "vedette".

Un autre mot du sicilien qui est impossible à rendre en français est strafalaria(20), qui n'a pas davantage d'équivalent en italien. Dans ce contexte, le terme doit être interprété dans son sens figuré de " femme de la rue et cancanière ". L'intention communicative du mot original, n'est pas transposée de façon équivalente dans la version française qui propose l'épithète, atténué, "saleté ". Le ton de la V0 est nettement affaibli dans la VD. De plus, dans la VO Grazia dit " vatinni " (" va-t-en ") à cette femme tandis que dans la VD " lâche-moi " pourrait suggérer un contact alors qu'il n'y en a pas.

Voici la scène en question:

\begin{tabular}{|l|l|}
\hline VO & VD \\
\hline Grazia : che vai dicendo? Testa di pane duro! & Grazia : qu'est-ce que tu racontes ? Tête de morue ! \\
\hline $\begin{array}{l}\text { Donna : testa di pani duru a mia? A mia testa di } \\
\text { pani duru? Ma chi ti credi di essere? Patty Pravo? }\end{array}$ & $\begin{array}{l}\text { Femme : Tête de morue moi ? Elle m'a dit " tête de } \\
\text { morue ? ". Tu te prends pour une vedette ou quoi ? }\end{array}$ \\
\hline Grazia : ma vatinni, strafalària! & Grazia : Lâche-moi, saleté ! \\
\hline
\end{tabular}

\section{- Macroscène 13, minute 45:20}

Marinella et le carabinier Pier Luigi cherchent un moment de tranquillité et de complicité près d'une petite colline mais ils sont dérangés par le frère cadet de la jeune fille, Filippo, qui essaye de toutes les manières possibles de ramener sa sœur à la maison :

\begin{tabular}{|l|l|}
\hline V0 & VD \\
\hline$[\ldots]$ & {$[\ldots]$} \\
\hline Carabiniere : non vedi che non vuole venire? & Carabinier : elle veut pas partir. \\
\hline
\end{tabular}

(20) Strafalàriu: le terme, attesté même dans sa variante strafallàriu, a différentes acceptions. II peut vouloir dire selon les cas : désordonné, distrait, extravagant, farfelu, vilain ; au sens figuré on le dit d'une femme de la rue, coquette, vieille commère cancanière et négligée. 
Traduction multimédia et voix régionales : la version française du film Respiro d'Emanuele Crialese

\begin{tabular}{|c|c|}
\hline V0 & VD \\
\hline Filippo : ma tu chi vo? & Filippo : qu'est-ce que t'as, toi? \\
\hline Marinella : Fili finiscila! Vatinni! & Marinella : Filippo, laisse-moi tranquille ! \\
\hline $\begin{array}{l}\text { Filippo : Cu ti canusci a tia ca parli? Cu ti canusci } \\
\text { a tia ca parli? ma si me soru ava a veniri cu mia? } \\
\text { Chi vo tuni? }\end{array}$ & $\begin{array}{l}\text { Filippo : tu te prends pour qui. T'es qui, toi? } \\
\text { C'est ma sœur ! Elle vient avec moi. Alors, } \\
\text { qu'est-ce que tu veux ? Qu'est-ce que tu veux? }\end{array}$ \\
\hline $\begin{array}{l}\text { Carabiniere : non ti capisco, non ti capisco quando } \\
\text { parli! Ma cosa ha detto? Cosa ha detto? }\end{array}$ & $\begin{array}{l}\text { Carabinier : elle veut pas rentrer à la maison } \\
\text { en ce moment! }\end{array}$ \\
\hline Filippo : co-co-co-co-co. lo manco ti capisco! & Filippo : espèce de poulet. ...co-co-co-co-co. \\
\hline Marinella : ancora? & Marinella : ça suffit. Arrête! \\
\hline$[\ldots]$ & {$[\ldots]$} \\
\hline Carabiniere : sei contento? & Carabinier : t'es content? \\
\hline Filippo : uhm, un parlari accussi picchi... & Filippo : ne parlez pas parce-que... \\
\hline Marinella : leviti vah, piffauri! & Marinella : pousse-toi! \\
\hline Filippo : buffia ca... cu si senti, Rambo? & Filippo : bouffon, va ! II se prend pour Rambo. \\
\hline Marinella : oh ma picchi un ti ni va tu? & Marinella : pourquoi t'y vas pas? \\
\hline Filippo : no & Filippo : non, je n'en ai pas envie! \\
\hline Marinella : ti stannu aspittannu, vatinni & $\begin{array}{l}\text { Marinella : ils t'attendent. Casse-toi, tu ne vois pas } \\
\text { qu'ils t'attendent? }\end{array}$ \\
\hline $\begin{array}{l}\text { Filippo : cu è chi m'aspetta? } \\
\text { Nun m'aspetta nuddu a mia }\end{array}$ & Filippo : personne m'attend! \\
\hline Carabiniere : vatinne... vatinne! & Carabinier : casse-toi ! \\
\hline $\begin{array}{l}\text { Filippo : comu? Vatinne? Ma chi dici? } \\
\text { Chi 'nchiappulia?? }\end{array}$ & $\begin{array}{l}\text { Filippo : mais qu'est-ce qu'il dit ? Trouduc! } \\
\text { C'est à moi que tu parles comme-ça? }\end{array}$ \\
\hline
\end{tabular}

Dans cette scène, le dialoguiste français a inséré encore une fois une trouvaille importante qui compense quelques-unes des pertes de la traduction : dans une des répliques entre Filippo et le carabinier, le garçon, qui bégaie, commence à répéter en italien la syllabe " $\mathrm{CO}$ " : " co-coco-co-co. lo manco ti capisco! ”. Dans la VD, le dialoguiste profite du bégaiement du jeune qui ressemble au début du cri du coq pour introduire dans la version française le mot d'argot " poulet " pour désigner le policier. Le dialoguiste est obligé de changer la réplique du carabinier qui, en italien, disait en parlant de Filippo : " non lo capisco " (je ne le comprends pas) car en effet Filippo parle un dialecte trop rapide, et donc incompréhensible. Dans la VD, le carabinier répète tout simplement " elle veut pas rentrer à la maison ". Toujours à propos de la difficulté de traduire les dialectes, notons dans cette même scène qu'au moment où le carabinier tente de parler en dialecte (vatinne), Filippo le ridiculise en lui jetant au visage l'expression sicilienne " chi 'nchiappulii "(21)? Dans la VD, cette phrase perd sa vivacité vernaculaire et est remplacée par une interjection vulgaire, " trouduc ", adressée au carabinier. Le français ne disposant d'aucune équivalence, c'est une sorte de compensation qui est opérée ici, en cohérence avec le reste des choix de traduction.

(21) Nchiappuliari : faire un mélange confus, faire quelque chose de pas clair, magouiller, ici plutôt baragouiner. 


\section{Conclusions}

Dans le domaine de la traduction pour le cinéma et par conséquent dans les pratiques du doublage et sous-titrage, la traduction doit être fonctionnelle (HOUSE, 1977 :103-109). Selon la théorie du Skopos (REISS 2000), le texte de départ cesse d'être l'élément central qui détermine la nature du texte d'arrivée et ce dernier devient une entité de plein droit dont la fonction peut être différente de celle du texte de départ. Par conséquent le traducteur-dialoguiste doit être libre de traduire le texte source à partir de tous les moyens que le texte cible met à sa disposition.

Selon Nida (1986, 1964 et 1969), la traduction consiste à " produire " dans la langue d'arrivée l'équivalent naturel le plus proche du message de la langue de départ, d'abord quant à la signification, puis quant au style. Le dialoguiste doit en conséquence rechercher la plus grande équivalence entre le parlé de l'original et le parlé de la VD dans un travail continu de négociation (PYM, 1992 : 27-39), entre échecs et réussites.

En général, le dialoguiste français a adopté une stratégie cibliste qui privilégie le spectateur de la langue d'arrivée afin de ne pas entraver la fluidité des dialogues du film en VD. Les adaptateurs ont transposé d'une manière adéquate, à notre avis, les moments où le film comprenait des passages vulgaires. La communication véhiculée par le doublage du sicilien au français est, somme toute adéquate (sauf quelques rares cas évoqués dans notre étude) et conserve les informations essentielles des contenus dialogiques de la VO.

En général, la VD garde un registre bas et familier et cherche à reproduire le ton de l'original, compte tenu de la difficulté incontournable du dialecte de l'île de Lampedusa (chaque personnage possédant un code intraduisible). La VO comprend, de plus, les intercalaires typiques de la langue parlée sicilienne qui ont souvent été omis en français dans la VD, sans grande perte de signification. La langue de la VD, en dépit de quelques trouvailles, est aplatie par la perte des nuances de changement des registres linguistiques : dans la VD, tous les personnages (sauf les enfants entre eux) parlent la même langue standardisée.

À notre avis, compte tenu de la nature et de la particularité du film Respiro, il aurait été préférable de ne pas faire de version doublée et de s'en tenir au sous-titrage(22). Le résultat de la VD n'est, par conséquent, pas toujours satisfaisant à cause de la contrainte majeure du dialecte parlé dans la VO. Malgré cela, ce film, grâce à son rythme et à son expressivité originale, a pu dépasser les frontières et avoir du succès en France.

antonino.velez@unipa.it

(22) Même un Italien qui n'est pas d'origine sicilienne ne peut suivre les dialogues de ce film qu'à l'aide des soustitres en italien. 


\section{Bibliographie}

BACCOLINI Raffaella, BOLLETTIERI BOSINELLI Rosa Maria, GAVIOLI Laura (eds.), 1994. I/ doppiaggio. Trasposizioni linguistiche e culturali, Clueb, Bologna, Italie.

BOLLETTIERI BOSINELLI Rosa Maria, HEISS Christine, SOFFRITTI Marcello, BERNARDINI Silvia, 2000. La traduzione multimediale. Quale traduzione per quale testo?, Clueb, Bologna, Italie.

CATFORD John C., 1965. A Linguistic Theory of Translation: an Essay on Applied Linguistics, Oxford University Press, London.

CIPOLLONI Marco, 1997. Lingue di celluloide. La traduzione del cinema in Italia. La Spagna e lo spagnolo nel cinema italiano e nel film multilingue, Edizioni dell'Orzo, Alessandri, Italie.

DELABASTITA Dirk, 1989. "Translation and mass-communication: film and TV. Translation as evidence of cultural dynamics ", Babel, 35, 4.

EVEN-ZOHAR, 1990. "The position of translated literature within the literary polysystem ", Poetics Today, n. 1, (11).

GENTZLER Edwin, 2001. Contemporary Translation Theories, Multilingual Matters Ltd.

GREGORY Michael, CARROL Susanne, 1978. Language and Situation. Language Varieties and Their Social Contexts, London, Routledge.

HERBST Thomas, 1994. Linguistiche Aspekte der Synchronisation von Fernsehserien. Phonetik, Textlinguistik, Übersetzungstheorie, Narr, Tübingen, Allemagne.

HERBST Thomas, 1996. "Why dubbing is impossible ". Traduzione Multimediale per il cinema, la televisione e la scena, (eds.) Christine Heiss et Rosa Maria Bollettieri Bosinelli, Bologna, Italie, CLUEB pp. 99-116.

HEWSON Lance, 2011. An Approach to Translation Criticism: "Emma " et "Madame Bovary " in Translation, J. Benjamins, Amsterdam, Pays-Bas, Philadelphia, États-Unis.

HOUSE Julian, 1977. " A model for translation quality assessment ", Meta, 22.

LE NOUVEL Thierry, 2007. Le doublage, Eyrolles, Paris.

MORINI Massimiliano, 2007. La traduzione. Teorie strumenti e pratiche, Sironi Editore, Milano, Italie.

NIDA Eugene, 1986. From One Language to Another. Functional Equivalence in Bible Translation, Thomas Nelson Publishers, Nashville, États-Unis.

NIDA Eugene, 1964. Toward a Science of Translating, E. H. Brill, Leiden, Pays-Bas.

NIDA Eugene, 1969. The Theory and Practice of Translation, E. H. Brill, Leiden, Pays-Bas. 
PAOLINELLI Mario, DI FORTUNATO Eleonora, 2005. Tradurre per il doppiaggio. Trasposizione linguistica dell'audiovisivo: teoria e pratica di un'arte imperfetta, Hoepli, Milano, Italie.

PAVESI Maria, 2005. La traduzione filmica. Aspetti del parlato doppiato dall'inglese all'italiano, Carocci, Roma.

PEREGO Elisa, 2005. La traduzione audiovisiva, Carocci, Roma.

PYM Antony, 1992. "Negotiation Theory as an Approach to Translation History. An Inductive Lesson from Fifteenth-Century Castile ", Yves Gambier and Jorma Tommola (eds.), Translation and Knowledge, 27-39, University of Turku - Centre for Translation and Interpreting, Turku, Finlande.

REISS Katharina, 2000. "Type, Kind and Individuality of Text. Decision Making in Translation ", The Translation Studies Reader, (ed.) Lawrence Venuti, Routledge, London.

SERROY Jean, 2006. Entre deux siècles. 20 ans de cinéma contemporain, Édition de la Martinière, Paris.

TOURY Gideon, 1995. Descriptive Translation Studies and Beyond, John Benjamins Publishing, Amsterdam.

VINAY Jean-Paul, DARBELNET Jean, 1968. Stylistique comparée du français et de l'anglais : Méthode de traduction, Didier, Paris.

Ancien élève de l'école d'interprètes de Trieste, Antonino Velez a été interprète de conférence et traducteur (spécialisé et littéraire) indépendant pour l'italien et le français. Depuis 2001, il enseigne la Linguistique française et la traductologie à I'Université de Palerme. II a été responsable de la filière de Tourisme (mention Tourisme culturel) de 2003 à 2010 de la faculté de lettres de l'Université de Palerme. Ses domaines de recherche sont : la linguistique française, la traductologie et la traduction, la traduction audiovisuelle et multimédia et la didactique des langues et de la traduction. En 2007, le ministère de l'Education nationale en France l'a nommé "Chevalier dans I'Ordre des Palmes Académiques ". II vient de publier une étude en français sur un des plus importants traducteurs contemporains français de l'italien : Bernard Simeone (Bernard Simeone traducteur de Luzi, Caproni et Sereni en France, Palermo, Herbita editrice, 2011). 